\title{
Oral Cancer: The Dentist's Intervention
}

\section{Shwetha Kumar, Kamlesh Dekate, Shilpa Patel, Nasreen Haque}

\begin{abstract}
Prevention and early detection are fundamental tools in the control of the oral cancer. Until recently screening and early detection of oral cancer has been dependent on a complete visual examination. Since simple examination is subject to observer variability adjunctive screening aids have been introduced to aid in early detection of oral cancer. The present paper outlines a brief overview of techniques in improving the identification of potentially malignant changes of the oral mucosa. We conclude that further research with clear objectives is strongly required to optimize and validate these oral screening procedures.
\end{abstract}

Keywords: Oral cancer, Premalignant lesion, Screening.

How to cite this article: Kumar S, Dekate K, Patel S, Haque N. Oral Cancer: The Dentist's Intervention. J Contemp Dent 2013; 3(2):74-77.

\section{Source of support: Nil}

Conflict of interest: None declared

\section{INTRODUCTION}

Oral cancer is a heterogeneous group of cancers arising from different parts of the oral cavity. It is the sixth most common cancer reported globally with an annual incidence of over 300,000 cases, of which $62 \%$ arise in developing countries. There is a significant difference in the incidence of oral cancer in different regions of the world. The incidence rates vary from over 20 per 100,000 population in India, to 10 per 100,000 in the US, and less than 2 per 100,000 in the middle East. In comparison with the US population, where oral cavity cancer represents only about 3\% of malignancies, it accounts for over $30 \%$ of all cancers in India. Despite advances in surgery, radiation and chemotherapy, the 5 years survival rate for oral cancer remains at about 57\%. ${ }^{1}$

\section{ETIOLOGY}

The strong association between cancers of the oral cavity and pharynx with tobacco use is well established. Tobacco has a distinct carcinogenicity - both as an initiator and promoter in head and neck cancers; alcohol and areca nut are proven carcinogens. The risks associated with smoking, chewing and alcohol consumption are synergistic. Viruses (EBV, HPV) are other important risk factors as are adjuvant carcinogens which act as distinct promotion factors viz poor dental hygiene for mouth cancer, vocal abuse in laryngeal cancer, Plummer-Vinson's syndrome in postcricoid cancer and chronic sinusitis in maxillary cancer. The geographical variations in incidence and mortality are indicative of the global differences in the prevalence of risk factors. ${ }^{2}$

Despite the fact that the oral cavity is accessible for visual examination and that oral cancer and premalignant lesions have well-defined clinical diagnostic features, oral cancers are typically detected in their advanced stages. In fact, in India, 60 to $80 \%$ of patients present with advanced disease as compared to $40 \%$ in developed countries. Dental professionals role in diagnosing, treatment and prevention of oral disease and promotion of oral health can be utilized in identification of these potentially malignant lesions. It is important to emphasize on the guidelines that the general dentist has to follow, to provide a significant contribution to the field of oral cancer prevention and therapy. ${ }^{3,4}$

\section{SCREENING}

\section{Routine Visual Examination}

'Screening' as the process by which a practitioner evaluates an asymptomatic patient to determine if he or she is 'likely' or 'unlikely' to have a potentially malignant or malignant lesion. An inspection of the oral cavity is often part of a physical examination in a dentist's or physician's office. Although physicians are more likely to provide risk-factor counseling (such as tobacco cessation), they are less likely than dentists to perform an oral cancer examination. It is therefore imperative for the dentist to be equipped with skills to examine for any potentially malignant disorders. ${ }^{4}$

A thorough health history review should yield information about the patient's tobacco and alcohol use, hospitalization history, surgery experience, dietary patterns, medication regimen and other illnesses must be elicited by the dentist. The dental practitioner should then conduct a visual and tactile examination (referred to as 'examination' throughout this report) to detect the presence of any oral abnormality which could be a potentially malignant or malignant lesion. ${ }^{5,6}$

\section{Performing the Examination}

- The mouth be examined with an external light source.

- Mouth mirror and tongue blade should be used for improved visualization.

- Before the examination, the patient should be asked to remove all dental appliances.

- When examining mucosal surfaces, it is important to gently dry those surfaces with a gauze or air syringe, so that color or texture changes will become more obvious. 
- Extraoral examination-observe face, head and neck noting any asymmetry, color change and growths.

- Conduct bilateral palpation to detect enlarged nodes.

- With patient's mouth partially open, inspect labial mucosa of maxillary and mandibular vestibules.

- Observe for changes in color, texture and any swelling or other abnormality.

- Begin perioral and intraoral soft tissue examination by assessing lips.

- With teeth in occlusion, assess the buccal and labial aspects of the gingiva and alveolar ridges going from maxillary right posterior to left posterior and then to mandibular gingiva.

- Assess palatal and lingual aspects with mouth open.

- With patient's tongue at rest and mouth partially open, inspect dorsum of tongue for any abnormalities including pattern of the papillae.

- The patient should protrude tongue and with a $2 \times 2$ gauze, the examiner should inspect from side to side to note any abnormalities.

- Can use a mouth mirror to inspect the right and lateral tongue margins.

- Should palpate tongue while in grasp have patient lift tongue to inspect ventral surface.

- With patient's mouth open, depress base of tongue with mouth mirror or tongue depressor to inspect tonsillar and oropharyngeal areas.

- At same time, inspect hard and soft palatal areas.

- Tongue still raised, inspect floor of the mouth for changes and abnormalities.

- Perform bimanual intraoral palpation on the floor of the mouth.

\section{Signs}

- Red lesions (erythroplakia)

- White lesions (leukoplakia)

- Red and white lesions (speckled leukoplakia; erythroplakia).

\section{Oral Lesions to look out for-Potentially Malignant Disorders ${ }^{7,8}$}

- Homogenous leukoplakia

- Nonhomogenous leukoplakia

- Erythroleukoplakia

- Submucous fibrosis.

\section{Tobacco-related Mucosal Alterations}

- Stomatitis nicotina

- Tobacco pouch keratosis.

\section{Oral Cancer Signs and Symptoms}

\section{Early}

- Sore in the mouth that does not heal (most common)

- White or red patch on gums, tongue, tonsil or soft tissue.

\section{Late}

- A lump or thickening in the cheek and/or neck

- A sore throat or a feeling that something is caught in the throat

- Difficulty in chewing or swallowing

- Difficulty in opening the mouth

- Difficulty in moving the jaw or tongue

- Numbness of the tongue or other area of the mouth

- Swelling of the jaw causing dentures to fit poorly

- Loosening of the teeth or pain around the teeth or jaw

- Voice changes

- Weight loss.

\section{ADJUNCTIVE SCREENING AIDS}

\section{Toluidine Blue}

Toluidine blue, a liquid dye composed of tolonium chloride, offers a potentially simple, inexpensive and sensitive chairside solution. This test is premised on the fact that mucosal cells with abnormal DNA, i.e. large nuclei, attract and retain the stain, even after the bulk of the stain has been washed off with acetic acid. The tissue that stains blue indicates either dysplasia or malignancy. ${ }^{9,10}$

\section{Chemiluminescence}

ViziLite Plus uses the principle of chemiluminescence which involves the use of a special light source most commonly a fluorescent light or a chemiluminescent light source and a blue dye (each system employs a 1\% acetic acid wash before use of its respective light source. Under blue-white illumination, abnormal squamous epithelium is reported to be distinctly white (acetowhite). ${ }^{11,12}$

\section{Autofluorescence}

VELscope takes advantage of the principle that each of our cells contain molecules capable of autofluorescence, especially when activated (excited) bispecific light waves. Excitation and emission of fluorescence depends on how light is scattered and absorbed in tissue: scattering is caused by differences in the index of refraction of different tissue components, while absorption is dependent on the molecular composition of the same components. An immature or dysplastic epithelial cell has abnormal composition as compared to a normal cell and so mucosal areas with such cells will not fluorescence, thereby appearing black..$^{13,14}$ 


\section{Transepithelial Cytology}

The oral brush biopsy, also known as OralCDx Brush Test system, involves a method of collecting and examining a transepithelial sample of cells from a mucosal lesion with appropriate representation of the superficial, intermediate and parabasal/basal layers of the epithelium. This method was specifically designed to investigate mucosal abnormalities that would otherwise not be subjected to biopsy because of low-risk clinical features. The brush biopsy is a painless procedure that captures the deeper epithelial cells on the bristles and the entire brush is sent to a pathology lab, where the cells are removed and plated on a microscopic slide. ${ }^{15}$

\section{Liquid-based Cytology}

Liquid-based cytology is an improvement in cytology technique that can compensate many disadvantages of conventional exfoliate cytology. This technique involves obtaining a suspension of cells is from which several slides could be prepared. The sample is immersed in a tube containing preservative fluid, which fixes the cells immediately and subjected to a centrifugal force. This results in the cells making a thin high cellular confined zone on the glass slide, which can be assessed easily. This method can be further used in combination with OralCDx Brush Test system. Instead of spreading the brush onto a glass slide, the brush is placed in the supplied glass tube, containing formalin $(10 \%)$ and placed in a vortex for 5 minutes in 4,000 rPM. This centrifugal force helped to sediment the cells and taken them off from the brush hairs. Then $100 \lambda\left(\mathrm{mm}^{3}\right)$ of this sediment was placed onto the cup of cytospine (Shandon UK) centrifuge in 1,000 rPM (similar to power recommended in this vortex for vaginal samples). Two to four samples were obtained from each cellular sediment. The more the sediment was rich in cellular material or blood component, the more glass slides were prepared. ${ }^{16,17}$

\section{Salivary Diagnostics}

Salivary diagnostics is a novel technique that uses saliva for disease diagnosis and health surveillance. In oral squamous cell carcinoma (OSCC), it is emerging as a newly developing and ongoing area of research that shows considerable promise as a noninvasive method for early detection of oral cancer. It is observed that proteins, such as mRNAs, enzymes and chemicals present in the saliva, are found sufficiently different in OSCC and normal controls, suggesting that these molecules might be potential biomarkers for OSCC. ${ }^{18}$ These potential salivary biomarkers for OSCC include:

1. Proteins of nuclear factor-kappa B (NF-אB) dependent cytokines [tumor necrosis factor (TNF), interleukin-1, interleukin-6, interleukin-8], basic fibroblast growth factor, Cyfra 21-1, cancer antigen-125 (CA-125), tissue polypeptide antigen, endothelin.

2. mRNA of interleukin-8, interleukin-1, DUSP1 (dual specificity phosphatase 1), H3F3A (H3 histone family 3A), OAZ1 (ornithine decarboxylase antizyme 1), S100P (S100 calcium binding protein P), SAT (spermidine/ spermine N1-acetyltransferase EST).

3. Reactive nitrogen species (nitric oxide and nitrates), GST (glutathione S-transferase), SOD (superoxide dismutase) and 8-OHdG (8-hydroxydeoxyguanosine). ${ }^{19}$

\section{In vivo Confocal Reflectance Microscopy}

Confocal microscopy was an imagining system originally used for visualization and study of ex vivo specimens but has been applied to in vivo human tissues in recent years. In vivo confocal reflectance microscopy has demonstrated the ability to obtain images of tissue architecture and cell morphology with resolution similar to histology. This technique utilizes an optical sectioning ability, resulting in no surgical procedure or histopathological sectioning and staining required for this procedure. Further studies are required to validate diagnostic accuracy of this technique and to consider it as vital noninvasive tool for the early detection of oral cancer and premalignant lesion. ${ }^{20}$

\section{CONCLUSION}

The ability to control oral and oropharyngeal cancer will depend on two salient factors: prevention and early diagnosis. A complete and thorough visual examination with the help of the aforementioned adjunctive techniques can aid in increasing our ability to differentiate between benign abnormalities and dysplastic changes as well as identify areas of dysplasia/early oral squamous cell carcinoma. With the lack of evidence to fully substantiate the use of screening programs for oral cancer, further studies using high-quality methodology on the use of oral cancer and prevention methods as well as the effectiveness of opportunistic screening in high risk groups are required. Continuing educational campaigns are needed on the local, state, and national level in order to educate the public about the risk factors and early signs/symptoms associated with this disease. Patients should to be encouraged to seek regular professional oral examinations by a dentist. Finally, dentists must be encouraged to perform oral cancer examinations 
as part of their patient care regime and to be knowledgeable about early signs of oral carcinoma.

\section{REFERENCES}

1. Vecchia LC, Tavani A, Franceshi S, Levi F, Corrao G, Negri E. Epidemology and prevention of oral cancer. Oral Oncol 1997 Sep;33(5):302-312.

2. Graham S, Dayal H, Rohrer T, Swanson M, Sultz H, Shedd D. Dentition, diet, tobacco and alcohol in the epidemiology of oral cancer. J Natl Cancer Inst 1977 Dec;59(6):1611-1618.

3. Wiwanitkit $\mathrm{S}$, Wiwanitkit V. Oral cancer screening. Indian J Community Med 2012 Jan-Mar;37(1):61.

4. Kujan O, Glenny AM, Duxbury J, Thakker N, Sloan P. Evaluation of screening strategies for improving oral cancer mortality: a Cochrane systematic review. J Dent Educ 2005 Feb;69(2):255-265.

5. Sankaranarayanan, Rengaswamy, et al. Early findings from a community-based, cluster-randomized controlled oral cancer screening trial in Kerala, India. Cancer 88.3(2000):664-673.

6. Lingen MW, Kalmar JR, Kerrison T, Speight PM. Critical evaluation of diagnostic aids for the detection of oral cancer. Oral Oncol 2008 Jan;44(1):10-22.

7. Bouquot JE, Farthing PM, Speight PM. The pathology of oral cancer and precancer. Curr Diagnos Path 2006 Sep;12(3):11-21.

8. Napier SS, Speight PM. Natural history of potentially malignant oral lesions and conditions: an overview of the literature. J Oral Pathol Med 2008 Jan;37(1):1-10.

9. Gray M, Gold L, Amanda Burls A, Elley K. The clinical effectiveness of toluidine blue dye as an adjunct to oral cancer screening in general dental practice. West Midlands DES report (England); 2000.

10. Epstein JB, Sciubba J, Silverman S Jr, Sroussi HY. Utility of toluidine blue in oral premalignant lesions and squamous cell carcinoma: continuing research and implications for clinical practice. Head Neck 2007 Oct;29(10):948-958.

11. Kerr AR, Sirois DA, Epstein JB. Clinical evaluation of chemiluminescent lighting: an adjunct for oral mucosal examinations. J Clin Dent 2006;17(3):59-63.

12. Oh ES, Laskin DM. Efcacy of the ViziLite system in the identification of oral lesions. J Oral Maxillofac Surg 2007 Mar;65(3):424-426.

13. Betz CS, Mehlmann M, Rick K, Stepp H, Grevers G, Baumgartner R, Leunig A. Autofluorescence imaging and spectroscopy of normal and malignant mucosa in patients with head and neck cancer. Lasers Surg Med 1999;25(4):323-334.
14. Svistun E, Alizadeh-Naderi R, El-Naggar A, Jacob R, Gillenwater A, Richards-Kortum R. Vision enhancement system for detection of oral cavity neoplasia based on autofluorescence. Head Neck 2004 Mar;26(3):205-215.

15. Sciubba JJ. Improving detection of precancerous and cancerous oral lesions. Computer-assisted analysis of the oral brush biopsy. US. Collaborative OralCDx Study Group. J Am Dent Assoc 2002 Oct;133(10):272-274.

16. Kujan O, Desai M, Sargent A, Bailey A, Turner A, Sloan P. Potential applications of oral brush cytology with liquid-based technology: results from a cohort of normal oral mucosa. Oral Oncol 2006 Sep;42(8):810-818.

17. Mozafari PM, Delavarian Z, Mohtasham N. Diagnostic aids in oral cancer screening. Oral Cancer 2012:189-208.

18. Nagler R, Bahar G, Shpitzer T, Feinmesser R. Concomitant analysis of salivary tumor markers-a new diagnostic tool for oral cancer. Clin Cancer Res 2006 Jul;12(13):3979-3984.

19. Cheng YSL, Wright J. Advances in diagnostic adjuncts for oral squamous cell carcinoma. Open Path J 2011;5:3-7.

20. Maitland KC, Gillenwater AM, Williams MD, El-Naggar AK, Descour MR, Richards-Kortum RR. In vivo imaging of oral neoplasia using a miniaturized fiber optic confocal reflectance microscope. Oral Oncol 2008 Nov;44(11):1059-1066.

\section{ABOUT THE AUTHORS}

\section{Shwetha Kumar (Corresponding Author)}

Senior Lecturer, Department of Oral Pathology, MGM Dental College and Hospital, Navi Mumbai, Maharashtra, India, Phone: 02227426604, e-mail: shwetha.vasanth@gmail.com

\section{Kamlesh Dekate}

Reader, Department of Oral Pathology, MGM Dental College and Hospital, Navi Mumbai, Maharashtra, India

\section{Shilpa Patel}

Professor and Head, Department of Oral Pathology, MGM Dental College and Hospital, Navi Mumbai, Maharashtra, India

\section{Nasreen Haque}

Tutor, Department of Oral Pathology, MGM Dental College and Hospital, Navi Mumbai, Maharashtra, India 